\title{
Pneumocystis pneumonia, a COVID-19 mimic, reminds us of the importance of HIV testing in COVID-19
}

\author{
Authors: Sophie Kelly, ${ }^{\mathrm{A}}$ Laura Waters, ${ }^{\mathrm{B}}$ Muge Cevik, ${ }^{\mathrm{C}}$ Simon Collins, ${ }^{\mathrm{D}}$ Joe Lewis, ${ }^{\mathrm{E}}$ Meng-San Wu, ${ }^{\mathrm{F}}$ Tom J Blanchard ${ }^{\mathrm{G}}$ \\ and Anna M Geretti ${ }^{\mathrm{H}}$
}

\begin{abstract}
While clinical environments are highly focused on COVID-19, reports of missed or delayed treatment for conditions that imitate COVID-19, such as pneumonia caused by the fungus Pneumocystis jirovecii, are emerging. Given the uncertain spectrum of COVID-19 presentations and variable sensitivity of laboratory tests for SARS-CoV-2, there is a risk that, without a high index of suspicion, alternative aetiologies may be overlooked while pursuing a diagnosis of COVID-19. The British HIV Association has been calling for the inclusion of HIV testing in all patients admitted to hospital with suspected COVID-19. In this article we reflect on the importance of including HIV testing to prevent avoidable morbidity and mortality in our patients.
\end{abstract}

KEYWORDS: HIV, COVID-19, Pneumocystis jirovecii, PCP, SARSCoV-2

DOI: $10.7861 /$ clinmed.2020-0565

\section{Case presentation}

In March 2020, a middle-aged man presented to community care in England with a 4-week history of shortness of breath, night sweats and weight loss. A chest X-ray was unremarkable, and he was prescribed oral antibiotics. A week later, he presented to emergency care. A chest X-ray showed bilateral apical ground glass changes (Fig 1a); he was clinically stable without significant hypoxia and was therefore discharged with a diagnosis of suspected mild COVID-19, in the absence of laboratory tests. Four

Authors: ${ }^{\text {A }}$ academic foundation doctor, University of Liverpool, Liverpool, UK and Royal Liverpool Hospital, Liverpool, UK; ${ }^{\text {B consultant }}$ in HIV medicine and chair of the British HIV Association, Mortimer Market Centre, London, UK; ${ }^{C}$ clinical academic fellow in infectious diseases, University of St Andrews, St Andrews, UK; ${ }^{\text {D }}$ co-founder, HIV i-Base, London, UK; ' Eacademic clinical lecturer in infectious diseases, University of Liverpool, Liverpool, UK and Royal Liverpool Hospital, Liverpool, UK; F core medical trainee, Royal Liverpool Hospital, Liverpool, UK; ${ }^{G}$ consultant in infectious diseases, University of Liverpool, Liverpool, UK and Royal Liverpool Hospital, Liverpool, UK; ${ }^{H}$ professor of virology and infectious diseases, University of Liverpool, Liverpool, UK and Royal Liverpool Hospital, Liverpool, UK days later, he presented again with significant hypoxia, worsening bilateral upper lobe airspace shadowing on chest X-ray (Fig 1b), anaemia (haemoglobin at $87 \mathrm{~g} / \mathrm{dL}$ ), lymphopenia $\left(0.14\right.$ cells $\left./ \mathrm{mm}^{3}\right)$ and raised C-reactive protein $(212 \mathrm{mg} / \mathrm{L})$; at this time oral thrush was noted.

\section{Diagnosis}

On first presentation to the emergency department, perhaps due to the relative frequency of COVID-19 during the peak of the pandemic and the consequent biases in clinical reasoning, COVID-19 was considered the most likely diagnosis. On second presentation, 4 days later, the diagnoses considered were community acquired pneumonia (CAP) and moderate/severe COVID-19. However, although the lymphopenia and bilateral chest X-ray changes could be consistent with COVID-19, the patient had some features that were not typical, such as oral thrush, associated weight loss and a prolonged clinical history. Pneumocystis jirovecii pneumonia (PCP) was first considered following admission and a negative SARS-CoV-2 polymerase chain reaction test $(P C R)$ result.

\section{Initial management and prognosis}

The patient was admitted and commenced on intravenous (IV) antibiotics for CAP. Naso-oropharyngeal swabs for SARS-CoV-2 PCR were negative on day 2 of admission. At this time, computed tomography (CT) of the chest and an HIV test were requested. The CT showed widespread ground glass changes with relative basal sparing (Figs 1c and d). The HIV test was positive. A presumptive diagnosis of PCP was made, but severe hypoxia contraindicated induced sputum for confirmation. He started IV co-trimoxazole (120 mg/kg) and oral prednisolone alongside high-flow oxygen. Tests indicated profound immunosuppression (CD4 count 28 cells $/ \mathrm{mm}^{3}$ ), a high plasma HIV-1 ribonucleic acid (RNA) load $(390,000$ copies $/ \mathrm{mL})$ and cytomegalovirus (CMV) viraemia (48,000 copies $/ \mathrm{mL}$ ). Blood tests for adenovirus deoxyribonucleic acid, toxoplasma immunoglobulin $\mathrm{G}$ and cryptococcal antigen, and urinary tests for legionella and pneumococcal antigens were negative.

\section{Case progression and outcome}

On admission day 5 , the patient deteriorated and was transferred to the intensive care unit for mechanical ventilation. An endotracheal 

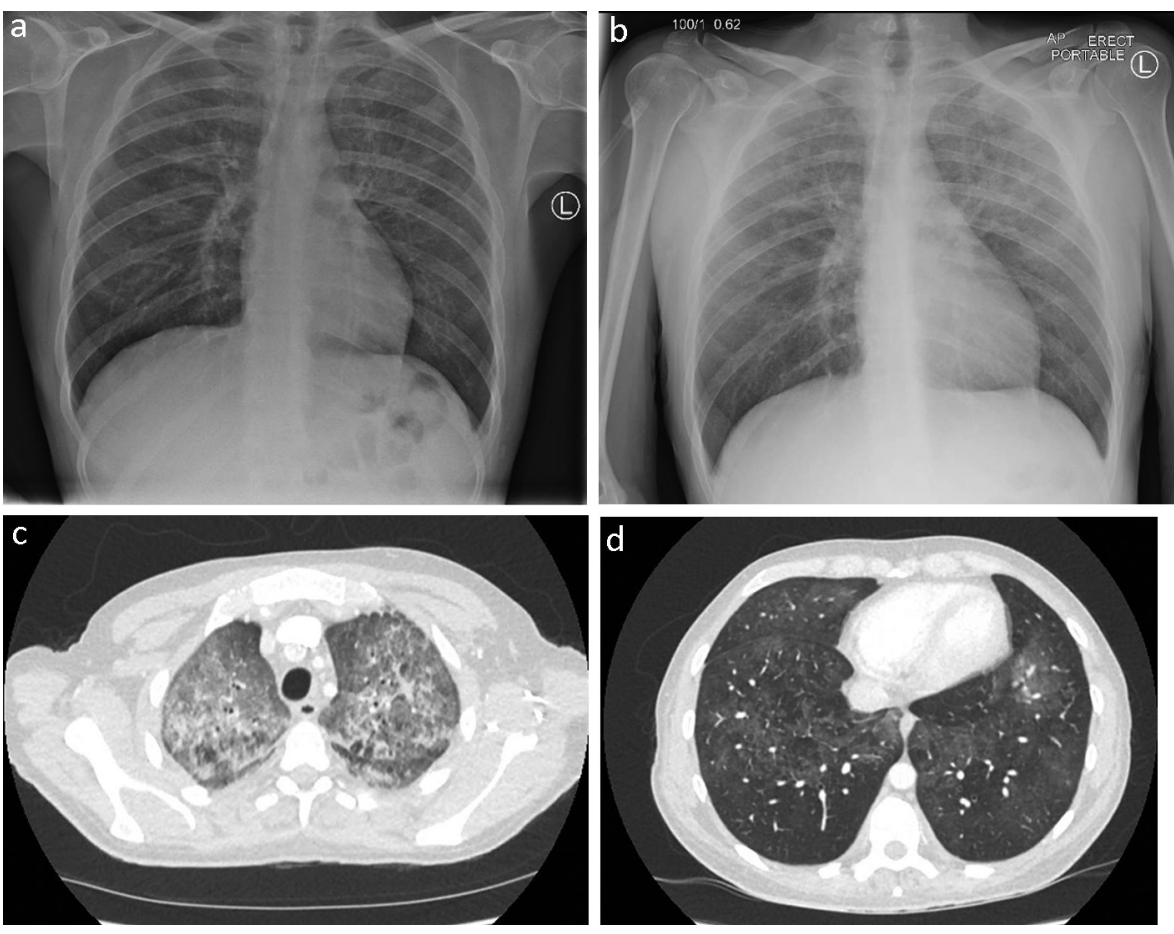

Fig 1. a) Chest X-ray showing bilateral apical ground glass changes. $b$ ) Chest $\mathrm{X}$-ray showing worsening bilateral upper lobe airspace shadowing. c and d) Computed tomography of the chest showing widespread ground glass changes with relative basal sparing.

aspirate tested negative for SARS-CoV-2, influenza, parainfluenza, rhinovirus, adenovirus, respiratory syncytial virus, human metapneumovirus and Pneumocystis jirovecii by PCR; acid-fast bacilli (AFB) staining was negative. On admission day 9 , due to bone marrow suppression and hyperkalaemia, co-trimoxazole was changed to IV pentamidine. On day 10, a bronchial alveolar lavage was positive for Pneumocystis jirovecii, thus confirming the diagnosis. The lavage was also positive for CMV and herpes simplex type 1 (HSV-1), but negative for SARS-CoV-2, respiratory viruses and AFB. A right tension pneumothorax and an intractable bronchopleural fistula developed on day 15 . The patient died on day 17 of hospital admission, approximately 29 days after initial presentation in the community.

\section{Discussion}

Reports of missed or delayed treatment for conditions that imitate COVID-19 such as pneumonia caused by the fungus Pneumocystis jirovecii are emerging. ${ }^{1,2}$ There is a risk that, without a high index of suspicion, alternative aetiologies may be overlooked while pursuing a diagnosis of COVID-19.

In England, there are an estimated 6,700 people living with undiagnosed HIV, of which 4,500 live outside London. ${ }^{3}$ About $24 \%$ of all new HIV diagnoses present with a CD 4 cell count $<200$ cells $/ \mathrm{mm}^{3}$ and this group is at high risk of AIDS-defining illness, most typically PCP. In people who present with PCP as the first manifestation of HIV infection, multiple factors influence disease outcomes, including timing of initiation of specific therapy. Overall survival for patients with PCP who require intensive care has improved over the past decades and survival rates are approximately $50 \% .^{4}$

Guidelines from the National Institute for Health and Care Excellence (NICE) recommend HIV testing in all hospital admissions for suspected CAP and in anyone with suspected PCP.
As these patients are now likely to come under the category of suspected COVID-19, the British HIV Association (BHIVA) has called for HIV testing of all suspected COVID-19 cases requiring hospitalisation. ${ }^{5}$ This is not an additional requirement where all emergency department attendees and hospital admissions are routinely screened for HIV. However, there remain settings where screening has not been adopted, in some cases despite estimated HIV prevalence rates $>2$ per 1,000 (in those aged 15-59 years), a threshold above which routine HIV testing is advised. Where the patient was admitted, there is no systematic HIV screening despite a regional prevalence of $\sim 2.2$ per 1,000 . At the same time, audit data demonstrate that among hospitalised patients with suspected COVID-19, the rate of HIV testing was only $5.5 \%$ $(140 / 2,529)$ in the period between 20 March 2020 and 21 April 2020. Perhaps more concerning, given recent case reports of co-infection with SARS-CoV-2 and Pneumocystis jirovecii, is that the rate was only $22 \%(15 / 69)$ in severe cases of PCR-confirmed COVID-19 requiring high-flow oxygen, non-invasive or invasive ventilation between 01 March 2020 and 18 May 2020.,2

\section{Summary}

In the case described, there were several clinical clues which could have triggered an earlier HIV test, including a longer clinical history prior to presentation to emergency care than typically reported for COVID-19, oral thrush and radiographical evidence of apical changes with basal sparing. One low-cost method of facilitating a differential diagnosis is to add a HIV test to the order panel for all COVID-19 admissions, and to educate and remain alert about the potential differential diagnoses, such as PCP. This will guide appropriate management for HIV-positive people with and without concomitant COVID-19, including appropriate cohort nursing and a targeted therapeutic plan. 


\section{Conflicts of interest}

Anna M Geretti has received consultancy fees and research funding from Gilead Sciences, ViiV Healthcare, and Janssen which are in relation to HIV. Anna M Geretti has also received fees and research funding from Roche Pharma Research and Early Development (pRED) outside of the work presented. Laura Waters reports advisory and speaker fees from Gilead, ViiV Healthcare, MSD, Janssen, Mylan and Cipla which are in relation to HIV.

\section{References}

1 Coleman H, Snell LB, Simons R, Douthwaite ST, Lee MJ. Coronavirus disease 2019 and Pneumocystis jirovecii pneumonia: a diagnostic dilemma in HIV. AIDS 2020;34:1258-60.

2 Mang S, Kaddu-Mulindwa D, Metz C et al. Pneumocystis Jirovecii Pneumonia and SARS-CoV-2 co-infection in newly diagnosed HIV-1 infection. Clin Infect Dis 2020:ciaa906 [Epub ahead of print].

3 O'Halloran C, Sun S, Nash S et al. HIV in the United Kingdom: Towards Zero 2030. 2019 report. London; Public Health England,
2019. https://assets.publishing.service.gov.uk/government/ uploads/system/uploads/attachment_data/file/858559/HIV_in_ the_UK_2019_towards_zero_HIV_transmissions_by_2030.pdf [Accessed 27 May 2020].

4 Miller RF, Allen E, Copas A, Singer M, Edwards SG. Improved survival for HIV infected patients with severe Pneumocystis jirovecii pneumonia is independent of highly active antiretroviral therapy. Thorax 2006;61:716-21.

5 Geretti AM, Collins S, Kelly S, Cevik M, Waters L. COVID-19 and HIV: Calling attention to the importance of ensuring HIV status and testing is included in the management of COVID-19. BMJ Blogs 07 April 2020. https://blogs.bmj.com/sti/2020/04/07/covid-19-andhiv-calling-attention-to-the-importance-of-ensuring-hiv-status-andtesting-is-included-in-the-management-of-covid-19 [Accessed 11 May 2020].

Address for correspondence: Prof Anna M Geretti, Ronald Ross Building, Institute of Infection, University of Liverpool, 8 West Derby Street, Liverpool L69 7BE, UK.

Email: geretti@liverpool.ac.uk

\section{CON/D) 19 and \\ A new Clinical Medicine and Future Healthcare Journal subject collection} bandemic manacement

Articles relating to the COVID-19 pandemic are now gathered together in one handy online subject collection for you to browse.

Browse the collection: www.rcpjournals.org/covid-19 